\title{
Effect of cutting frequencies and nitrogen levels on growth, green and seed yield and quality of water spinach (Ipomoea reptans Poir.)
}

\author{
R. K. Sarkar ${ }^{1}$, J. C. Jana ${ }^{2}$ and S. Datta ${ }^{3 *}$ \\ ${ }^{1}$ Darjeeling Krishi Vigyan Kendra, Uttar Banga Krishi Viswavidyalaya, Kalimpong, Darjeeling, INDIA \\ ${ }^{2}$ Department of Vegetable and Spice Crops, Uttar Banga Krishi Viswavidyalaya Pundibari, Cooch Behar- 736165 \\ (W. B.), INDIA \\ ${ }^{3}$ R.R.S., Terai zone Uttar Banga Krishi Viswavidyalaya Pundibari, Cooch Behar- 736165 (W.B.), INDIA \\ *Corresponding author. E-mail: suchanddatta@rediffmail.com
}

Received: July 11, 2014; Revised received: September 01, 2014; Accepted:. October 25, 2014

\begin{abstract}
To study the effect of cutting frequencies and nitrogen levels on growth, green and seed yield and quality of upland water spinach (Ipomoea reptans Poir.) an experiment was undertaken with four cutting frequencies $\left(\mathrm{C}_{0}=\right.$ no cutting; $C_{1}=$ one cutting at 45 days after sowing (DAS); $C_{2}=$ two cutting at 45 and 65 DAS and $C_{3}=$ three cutting at 45, 65 and 85 DAS) and five nitrogen levels ( $N_{0}=$ no application; $N_{1}=50 \mathrm{~kg} / \mathrm{ha} ; N_{2}=100 \mathrm{~kg} / \mathrm{ha} ; N_{3}=150 \mathrm{~kg} / \mathrm{ha}$ and $N_{4}=200 \mathrm{~kg} / \mathrm{ha}$ ). Results revealed that all growth, seed yield and seed quality attributes were found to decrease significantly with higher cutting frequencies, whereas, reverse effects on all physiological attributes, green yield and its quality parameters. On the other hand, nitrogen level (upto $150 \mathrm{~kg} \mathrm{~N} / \mathrm{ha}$ ) had significant effect and all these parameters. The highest green yield was recorded at three cuttings and $150 \mathrm{~kg} \mathrm{~N} / \mathrm{ha}$ individually and also at their interaction (16.34, 12.57 and $17.77 \mathrm{t} / \mathrm{ha}$, respectively). Maximum value of quality parameter like vitamin $\mathrm{A}$ (3072.9 $\mu \mathrm{g} / 100 \mathrm{~g}$ ) was recorded in one cutting with $150 \mathrm{~kg} \mathrm{~N} /$ hectare, whereas, maximum ascorbic acid was recorded maximum $(45.31 \mathrm{mg} / 100 \mathrm{~g})$ in one cutting without $\mathrm{N}$ fertilizer application Maximum net profit of Rs. 114324.00 with B: $C$ ratio of $2.22: 1$ was obtained with combination of three cuttings and $150 \mathrm{~kg} \mathrm{~N} / \mathrm{ha}$ and that may be adopted for its commercial cultivation in medium to upland situation under terai region of West Bengal.
\end{abstract}

Keywords: Cutting frequency, Growth, Ipomoea reptans, Nitrogen, quality, Water spinach, Yield

\section{INTRODUCTION}

Water spinach (Ipomoea reptans Poir.) is an under-exploited herbaceous perennial, leafy vegetable of the tropics and subtropics. It is also known as water convolvulus, swamp cabbage etc. is of East Indian or Chinese origin and belongs to the family convolvulaceae (morning glory) family. The edible portion contain up to $29 \%$ crude protein on a dry matter basis and is also rich in minerals and vitamins, being especially rich in vitamins A (carotene $2.9 \mathrm{~g} / 100$ $\mathrm{g}$ edible portion), $\mathrm{B}_{1}, \mathrm{~B}_{2}$ and $\mathrm{C}$ (45 mg/100 $\mathrm{g}$ edible portion) and iron. There are two main cultivar groups of var. aquatica and var. reptans. The first is an aquatic plant, propagated by cuttings and growing in the wild or cultivated in the fish ponds and water courses. The second is an upland vegetable and propagated mainly by seeds or cuttings (Palada and Crossman, 1999). A white flower is produced which matures into a four seeded pod. Flowering occurs under short day condition. But the cultivation of upland water spinach (I. reptans) is limited due to non-availability of seeds to the farmers. Nitrogen is one of the inevitable major nutrients and an indispensable constituent of protein and nucleic acid molecules. Nitrogen fertilization has become the key inputs in food production. It is essential for vegetative growth of the plant resulting in higher green and seed yield (Tehelan and Thakral, 2008; Tunctruk et al., 2011). Increased addition of nitrogen usually results in increased yield of crop plant (Korus and Lisiewska, 2009). Application of nitrogen to increase yield in leafy vegetables is a well recognized practice. Nitrogen deficiency exerts its effect on plant growth through reduced leaf area index and hence low light interception and low dry matter production (Masinde and Agong, 2012). Cutting management is found beneficial for improving green yield, number of branches, number of leaves, dry weight of leaves and stem, whereas, reverse effect is observed with some parameters like length of leaves, breadth of leaves and vine length (Thapa and Maity, 2004). Stem cutting significantly hastened flowering and enhanced branching (Ahmed and Oladiaran, 2012). As a result, green yield and seed yield increases by adopting proper cutting management of the crop. Since, most of the leafy vegetables several cuttings are possible, they require a good amount of nitrogenous fertilizer for their quick growth, good vegetative growth and as well 
as for reproductive growth (Jana et al., 1999; Datta et al., 2005; Datta et al., 2008). Information on green and seed yield of water spinach as affected by cutting frequencies and different levels of nitrogen for the terai zone of the West Bengal is meager. Hence, this experiment was undertaken.

\section{MATERIALS AND METHODS}

The present investigation was carried out during kharif seasons of 2007-08 and 2008-09 at the Instructional Farm of Uttar Banga Krishi Viswavidyalaya in terai region of West Bengal. The experimental field was medium low in position, sandy loam in texture, acidic in nature and with low fertility status. The sowing of seed was done directly in the main field in $1^{\text {st }}$ day of June and plot size was $4.5 \mathrm{sq}$ m with spacing of $30 \mathrm{~cm}$ $\times 15 \mathrm{~cm}$ in each experimental plot of 4.50 square meter area. The crop was supplied with 20 tonnes well rotten farmyard manure per hectare along with $60 \mathrm{~kg} \mathrm{P}_{2} \mathrm{O}_{5}$ and $80 \mathrm{~kg} \mathrm{~K} \mathrm{~K}_{2} \mathrm{\textrm {kg }}$ per hectare. Full amount of $\mathrm{P}_{2} \mathrm{O}_{5}$, $\mathrm{K}_{2} \mathrm{O}$ and $1 / 4^{\text {th }} \mathrm{N}$ as per treatment were applied at the time of land preparation and another $3 / 4^{\text {th }}$ of $\mathrm{N}$ in three equal splits as top dressing. The experiment was conducted with upland water spinach (I. reptans) with its locally collected genotype named as Kalmi Sag. The experiment was comprised of four cutting frequencies $\left(\mathrm{C}_{0}=\right.$ no cutting; $\mathrm{C}_{1}=$ one cutting at 45 days after planting; $\mathrm{C}_{2}=$ two cutting at 45 and 65 DAS and $\mathrm{C}_{3}=$ three cutting at 45,65 and 85 DAS) and five nitrogen levels $\left(\mathrm{N}_{0}=\right.$ no application; $\mathrm{N}_{1}=50 \mathrm{~kg} / \mathrm{ha} ; \mathrm{N}_{2}$ $=100 \mathrm{~kg} / \mathrm{ha} ; \mathrm{N}_{3}=150 \mathrm{~kg} / \mathrm{ha}$ and $\mathrm{N}_{4}=200 \mathrm{~kg} / \mathrm{ha}$ ) and was conducted in factorial randomized block design with three replications. Harvesting of green leaves with tender stems was done by top shoot cutting of 25-30 $\mathrm{cm}$ length twigs leaving $10 \mathrm{~cm}$ above the ground. Thereafter, subsequent harvesting was done at 20 days interval. After 3 times harvesting of green twigs, the plants were left for seed production. Flowering generally starts when the day become shorter and average temperature gradually goes down from October and seed sets in December. Harvesting of fruits was done by picking with hands in the month of middle of February when fruits turned to yellow colour. The harvested fruits were dried on threshing floor and then seeds were extracted plot wise separately. Chlorophyll content of green leaves was recorded at time flowering by chlorophyll meter. Leaf area index and crop growth rate was estimated. Ascorbic acid content in water spinach was determined by colorimetric method based on the reduction of 2, 6-dichlorophenol indophenol by ascorbic acid and was expressed in milligram of ascorbic acid per $100 \mathrm{~g}$ of sample (Ranganna, 2001). Vitamin A content of leaf was estimated by analyzing the beta carotene content of the leaf and dividing the value with 0.6 to obtain the vitamin A content of leaf (Ranganna, 2001). Different seed testing parameters like shelling percentage, test weight, germination percentage, seedling vigour index
(SVI) and seedling growth rate was calculated. The statistical analysis of data was done as per method suggested by Gomez and Gomez (1984). Then the weight of the cleaned and dried seeds from each of the experimental plots as per treatment schedule was recorded for computation of seed yield. All the seed quality testing parameters was carried out in the month of May. The cost of cultivation and gross return were estimated on the basis of price of different components fixed by the state Government to work out the economics.

\section{RESULTS AND DISCUSSION}

In the present study different plant growth attributes viz. vine length, number of nodes/plant and average internodes length gave significant changes in different cutting frequency and nitrogen levels (Table 1). The maximum vine length $(44.30 \mathrm{~cm})$, number of nodes/ plant (22.68) and average internodes length $(1.68 \mathrm{~cm})$ in control treatment $\left(\mathrm{C}_{0}\right)$, whereas, they were reported to be minimum at highest cutting frequency i.e. three cuttings $\left(\mathrm{C}_{3}\right)$. It might be due to the apical dominance which causes development of lateral buds, ultimately increase the number of shoots. Reduction in vine length by taking three cuttings $\left(\mathrm{C}_{3}\right)$ was $53.76 \%$ as compared to control $\left(\mathrm{C}_{0}\right)$. Thapa and Maity (2004) reported that cutting management significantly reduced the vine length, length of leaves, etc. These parameters were significantly increased with greater supply of nitrogen upto $150 \mathrm{~kg} \mathrm{~N} / \mathrm{ha}$, thereafter, decreased at highest N level. Significantly maximum vine length $(35.42 \mathrm{~cm})$, number of nodes/plant (19.98) and average internodes length $(1.79 \mathrm{~cm})$ were recorded as a result of higher nitrogen level of $150 \mathrm{~kg} \mathrm{~N} / \mathrm{ha}\left(\mathrm{N}_{3}\right)$ and this was statistically at par with $200 \mathrm{~kg} \mathrm{~N} / \mathrm{ha}\left(\mathrm{N}_{4}\right)$. Li and Harahap (1994) reported that nitrogen fertilizer application led to a significant increase of plant height, number of nodes, stem diameter and average intermodal length in water spinach which are in conformity with the present investigation. The improvement in all growth parameters with higher nitrogen fertilizer application might be due to the contribution of nitrogen to its role in plant growth, photosynthesis and synthesis of proteins. Interaction between cutting frequency and nitrogen levels was found to be significant.

All the physiological parameters viz. leaf area index, crop growth rate and leaf chlorophyll content (Table 1) which are very important yield attributing characters, were significantly influenced due to cutting frequency as well as nitrogen application. Among cutting treatments, significantly maximum (45.54 SPAD 502) leaf chlorophyll was recorded in one cutting $\left(\mathrm{C}_{1}\right)$. This was statistically at par with two cuttings $\left(\mathrm{C}_{2}\right)$ treatment (45.10 SPAD 502), while, it was found minimum (41.49 SPAD 502) at control $\left(\mathrm{C}_{0}\right)$. Besides, LAI (1.24) and CGR $\left(6.62 \mathrm{~g} / \mathrm{m}^{2} /\right.$ day $)$ were also found to be maximum with three cuttings $\left(\mathrm{C}_{3}\right)$ closely followed by 
two cuttings $\left(\mathrm{C}_{2}\right)$ and minimum (LAI, 0.88 and CGR, $5.18 \mathrm{~g} / \mathrm{m}^{2} /$ day) at control $\left(\mathrm{C}_{0}\right)$. Significant variations were reported by application of nitrogen fertilizer with respect to all these parameters. The application $200 \mathrm{~kg}$ $\mathrm{N} / \mathrm{ha}\left(\mathrm{N}_{4}\right)$ recorded maximum (47.23 SPAD 502) chlorophyll and minimum (39.37 SPAD 502) at control $\left(\mathrm{N}_{0}\right)$. Significantly maximum LAI (1.29) and CGR $\left(6.46 \mathrm{~g} / \mathrm{m}^{2} /\right.$ day $)$ were recorded as a result of application of $150 \mathrm{~kg} \mathrm{~N} / \mathrm{ha}\left(\mathrm{N}_{3}\right)$ followed by $\mathrm{N}_{4}$ and $\mathrm{N}_{2}$ treatments. The control treatment $\left(\mathrm{N}_{0}\right)$ gave minimum values (LAI, 0.81 and CGR, $5.02 \mathrm{~g} / \mathrm{m}^{2} /$ day) of these parameters. The increase in LAI in response to an increase in $\mathrm{N}$ fertilizer is probably due to enhanced availability of nitrogen which enhanced more leaf area resulting in higher photo assimilates and thereby in more dry matter accumulation. Interaction between cutting frequency and nitrogen had positive effect on all physiological attributes. Three cuttings with $150 \mathrm{~kg}$ N/ha $\left(\mathrm{C}_{3} \mathrm{~N}_{3}\right)$ recorded maximum LAI (1.49) and CGR $\left(7.19 \mathrm{~g} / \mathrm{m}^{2} /\right.$ day), whereas, the leaf chlorophyll was found to be maximum (49.00 DPAD 502) in $\mathrm{C}_{1} \mathrm{~N}_{4}$ combination. All these attributes were minimum in control $\left(\mathrm{C}_{0} \mathrm{~N}_{0}\right)$. The highest leaf chlorophyll under highest $\mathrm{N}$ level might be due to maximum instant nitrogen could have absorbed and used which could lead to increase in chlorophyll content. Singh et al. (1986) reported that nitrogen application increased leaf chlorophyll content in amaranthus.

The perusal of data (Table 1) revealed that green yield of water spinach significantly increased with higher cutting frequencies and also due to higher nitrogen level upto application of $150 \mathrm{~kg} \mathrm{~N} / \mathrm{ha}$, thereafter, decreased at $200 \mathrm{~kg} \mathrm{~N} / \mathrm{ha}$. Significantly highest green yield (16.34 t/ha) was recorded at three cuttings $\left(\mathrm{C}_{3}\right)$, the second highest (10.66 t/ha) was obtained by two cutting $\left(\mathrm{C}_{2}\right)$. The lowest green yield of $5.85 \mathrm{t} / \mathrm{ha}$ was observed in one cutting $\left(\mathrm{C}_{1}\right)$. Jana et al. (1999) reported the maximum green leaf yield of palak with three cuttings which are in accordance with present study. On the other hand, significantly highest green yield $(12.57 \mathrm{t} / \mathrm{ha})$ was registered with the application of $150 \mathrm{~kg} \mathrm{~N} / \mathrm{ha}\left(\mathrm{N}_{3}\right)$ and lowest green yield of $9.27 \mathrm{t} / \mathrm{ha}$ was obtained in control $\left(\mathrm{N}_{0}\right)$. The green yield increased with higher $\mathrm{N}$ fertilizer application rate might be due to better nutritional environment in the root zone as well as in the plant system. The combination of three cuttings with $150 \mathrm{~kg} \mathrm{~N} / \mathrm{ha}\left(\mathrm{C}_{3} \mathrm{~N}_{3}\right)$ gave significantly highest green yield (17.77 t/ha) which was statistically at par with $\mathrm{C}_{3} \mathrm{~N}_{4}(17.29 \mathrm{t} / \mathrm{ha})$. Datta et al., (2008) reported that green leaf yield was significantly increased with the increase in nitrogen levels in coriander.

The quality of green produce viz. ascorbic acid, vitamin $\mathrm{A}$ and leaf nitrate content (Table 1) were significantly affected by cutting frequency and nitrogen levels. Significantly maximum ascorbic acid $(43.02 \mathrm{mg} / 100 \mathrm{~g})$, vitamin A $(2802.4 \mu \mathrm{g} / 100 \mathrm{~g})$ and leaf nitrate content $(401.0 \mathrm{mg} / \mathrm{kg})$ were recorded in one cutting $\left(\mathrm{C}_{1}\right)$ closely followed by two cuttings $\left(\mathrm{C}_{2}\right)$, whereas, all these parameters were found to be minimum at control treatment $\left(\mathrm{C}_{0}\right)$. Tomar (2001) reported that all green quality parameters such as ascorbic acid and vitamin A were significantly influenced with cutting management in spinach. The application of $150 \mathrm{~kg} \mathrm{~N} / \mathrm{ha}$ indicated significant increase in the quality of water spinach leaf with respect to vitamin A (26.4\%) and leaf nitrate $(25.8 \%)$ higher over control $\left(\mathrm{N}_{0}\right)$. The greater nitrogen resulted more vitamin A synthesis. It might be due to the participation of nitrogen in carotene synthesis which is the precursor of vitamin A. Reverse effect was noticed in ascorbic acid due to increase in $\mathrm{N}$ levels and maximum $(44.68 \mathrm{mg} / 100 \mathrm{~g})$ was recorded in control $\left(\mathrm{N}_{0}\right)$. This might be due to the interaction of nitrogen in the hormonal metabolism of the plant system, especially the auxins. Interaction between cutting frequency and nitrogen level showed positive results on all green quality attributes. Singh et al. (1986) reported that nitrogen increased crude protein and carotene content but decreased the ascorbic acid content in amaranthus which are in good connection with the findings of present study. The leaf nitrate accumulated due to all the treatments remains within the daily acceptable limit. On the other hand, Schmidt et al. (2007) reported the similar findings in different leafy vegetable including water spinach (average nitrate content of $0.39 \%$ ) which are in accordance with the present investigation in respect to leaf nitrate content.

The depicted results on seed yield and its attributes viz. number of flowers/hill, number of capsules/hill and seed yield (Table 2) were significantly reduced due to higher cutting frequency but reverse effects were observed in all these parameters to be higher with greater nitrogen supply upto $150 \mathrm{~kg} \mathrm{~N} / \mathrm{ha}$, thereafter, decreased at $200 \mathrm{~kg} \mathrm{~N} / \mathrm{ha}$ (Table 2). Significantly maximum number of flowers/hill (274.3), number of capsules/hill (210.6) and seed yield (1.17 t/ha) were recorded under no cutting $\left(\mathrm{C}_{0}\right)$, whereas, all these were lowest at three cutting $\left(C_{3}\right)$. Jana et al. (1999) reported that no cutting gave the highest seed yield in palak. The application of $150 \mathrm{kgN} / \mathrm{ha}\left(\mathrm{N}_{3}\right)$ significantly increase, $30.80 \%, 55.33 \%$ and $36.0 \%$ higher number of flowers/hill, number of capsules/hill and seed yield, respectively, as compared to control $\left(\mathrm{N}_{0}\right)$. Interaction between cutting frequency and $\mathrm{N}$ levels had linear effects with respect to these attributes. The combination of no cutting and $150 \mathrm{~kg} \mathrm{~N} / \mathrm{ha}\left(\mathrm{C}_{0} \mathrm{~N}_{3}\right)$ recorded maximum number of flowers/hill (308.6), number of capsules/hill (249.5) and seed yield (1.32 t/ha) closely followed by $\mathrm{C}_{0} \mathrm{~N}_{4}, \mathrm{C}_{1} \mathrm{~N}_{3}$ and $\mathrm{C}_{0} \mathrm{~N}_{2}$, whereas, combination of $\mathrm{C}_{3} \mathrm{~N}_{0}$ produced minimum values all these traits. Datta et al. (2008) also reported significant increase in different yield attributing characters and seed yield with an increase in nitrogen levels in coriander which were in good connection with the 
Table 1. Effect of cutting frequencies and nitrogen levels on growth, physiological, green yield and its quality parameters of water spinach (pooled).

\begin{tabular}{|c|c|c|c|c|c|c|c|c|c|c|}
\hline $\begin{array}{l}\text { Treat- } \\
\text { ment }\end{array}$ & $\begin{array}{c}\text { Vine } \\
\text { length } \\
(\mathrm{cm})\end{array}$ & $\begin{array}{c}\text { No. of } \\
\text { nodes } \\
/ \\
\text { plant } \\
\end{array}$ & $\begin{array}{c}\text { Avg. } \\
\text { internodes } \\
\text { length } \\
\text { (cm) } \\
\end{array}$ & $\begin{array}{c}\text { Leaf } \\
\text { area } \\
\text { index }\end{array}$ & $\begin{array}{c}\text { Crop } \\
\text { growth } \\
\text { rate }\end{array}$ & $\begin{array}{c}\text { Chlorophyll } \\
\text { content } \\
\text { (SPAD 502) }\end{array}$ & $\begin{array}{c}\text { Green } \\
\text { yield } \\
(\mathrm{t} / \mathrm{ha})\end{array}$ & $\begin{array}{l}\text { Ascorbic } \\
\text { acid } \\
(\mathrm{mg} / \mathbf{1 0 0 g})\end{array}$ & $\begin{array}{c}\text { Vitamin } \\
\text { A }(\mu \mathrm{g} / \\
100 \mathrm{~g})\end{array}$ & $\begin{array}{l}\text { Nitrate } \\
\text { content } \\
(\mathrm{mg} / \mathrm{kg})\end{array}$ \\
\hline \multicolumn{11}{|c|}{ Cutting frequency } \\
\hline $\mathrm{C}_{0}$ & 44.30 & 22.68 & 1.68 & 0.88 & 5.18 & 41.49 & 0.00 & 40.98 & 2649.4 & 369.0 \\
\hline $\mathrm{C}_{1}$ & 31.50 & 18.58 & 1.67 & 1.03 & 5.64 & 45.54 & 5.85 & 43.02 & 2802.4 & 401.0 \\
\hline $\mathrm{C}_{2}$ & 29.35 & 16.77 & 1.64 & 1.13 & 6.13 & 45.10 & 10.66 & 42.36 & 2759.9 & 395.0 \\
\hline $\mathrm{C}_{3}$ & 28.81 & 16.31 & 1.61 & 1.24 & 6.62 & 43.37 & 16.34 & 41.83 & 2716.8 & 388.0 \\
\hline S. Em \pm & 0.19 & 0.13 & 0.01 & 0.01 & 0.01 & 0.15 & 0.08 & 0.16 & 10.62 & 2.40 \\
\hline $\begin{array}{l}\text { C.D. at } \\
5 \%\end{array}$ & 0.54 & 0.37 & 0.04 & 0.02 & 0.03 & 0.44 & 0.24 & 0.67 & 29.81 & 6.70 \\
\hline \multicolumn{11}{|c|}{ Nitrogen level } \\
\hline $\mathrm{N}_{0}$ & 30.95 & 17.10 & 1.47 & 0.81 & 5.02 & 39.37 & 9.27 & 44.68 & 2375.3 & 337.0 \\
\hline $\mathrm{N}_{1}$ & 32.51 & 17.96 & 1.57 & 0.92 & 5.47 & 42.79 & 10.12 & 43.38 & 2561.8 & 364.0 \\
\hline $\mathrm{N}_{2}$ & 33.90 & 18.61 & 1.67 & 1.14 & 6.17 & 44.57 & 10.96 & 41.65 & 2757.8 & 402.0 \\
\hline $\mathrm{N}_{3}$ & 35.42 & 19.98 & 1.79 & 1.29 & 6.46 & 45.41 & 12.57 & 40.55 & 3002.8 & 424.0 \\
\hline $\mathrm{N}_{4}$ & 34.67 & 19.28 & 1.74 & 1.20 & 6.34 & 47.23 & 11.84 & 39.98 & 2963.0 & 414.0 \\
\hline S. Em \pm & 0.22 & 0.15 & 0.01 & 0.01 & 0.01 & 0.17 & 0.09 & 0.18 & 11.87 & 2.70 \\
\hline $\begin{array}{l}\text { C.D. at } \\
5 \%\end{array}$ & 0.78 & 0.42 & 0.05 & 0.02 & 0.03 & 0.47 & 0.27 & 0.70 & 33.32 & 7.50 \\
\hline \multicolumn{11}{|c|}{ Interaction (C X N) } \\
\hline $\mathrm{N}_{0} \mathrm{C}_{0}$ & 42.55 & 21.08 & 1.48 & 0.67 & 4.37 & 37.79 & 0.00 & 43.52 & 2297.8 & 330.0 \\
\hline $\mathrm{N}_{0} \mathrm{C}_{1}$ & 28.30 & 17.01 & 1.50 & 0.78 & 4.91 & 40.78 & 4.29 & 45.31 & 2482.8 & 343.0 \\
\hline $\mathrm{N}_{0} \mathrm{C}_{2}$ & 26.89 & 15.32 & 1.48 & 0.86 & 5.19 & 40.10 & 8.69 & 45.18 & 2382.5 & 340.0 \\
\hline $\mathrm{N}_{0} \mathrm{C}_{3}$ & 26.07 & 14.98 & 1.45 & 0.93 & 5.63 & 38.82 & 14.83 & 44.72 & 2337.9 & 335.0 \\
\hline $\mathrm{N}_{1} \mathrm{C}_{0}$ & 43.70 & 22.22 & 16.2 & 0.75 & 4.79 & 40.27 & 0.00 & 42.28 & 2498.0 & 352.0 \\
\hline $\mathrm{N}_{1} \mathrm{C}_{1}$ & 30.23 & 17.93 & 1.59 & 0.88 & 5.43 & 44.36 & 4.92 & 44.51 & 2623.0 & 376. \\
\hline $\mathrm{N}_{1} \mathrm{C}_{2}$ & 28.30 & 16.02 & 1.55 & 0.97 & 5.53 & 44.25 & 9.87 & 43.73 & 2585.4 & 368.0 \\
\hline $\mathrm{N}_{1} \mathrm{C}_{3}$ & 27.83 & 15.68 & 1.53 & 1.07 & 6.14 & 42.28 & 15.57 & 43.01 & 254.8 & 362.0 \\
\hline $\mathrm{N}_{2} \mathrm{C}_{0}$ & 44.35 & 22.76 & 1.73 & 0.93 & 5.48 & 41.91 & 0.0 & 40.42 & 2703.3 & 381.0 \\
\hline $\mathrm{N}_{2} \mathrm{C}_{1}$ & 32.23 & 18.18 & 1.69 & 1.09 & 5.68 & 46.49 & 5.79 & 43.04 & 2799.7 & 415.0 \\
\hline $\mathrm{N}_{2} \mathrm{C}_{2}$ & 29.98 & 16.61 & 1.64 & 1.20 & 6.52 & 45.94 & 10.82 & 41.95 & 2774.9 & 411.0 \\
\hline $\mathrm{N}_{2} \mathrm{C}_{3}$ & 29.03 & 16.19 & 1.63 & 1.32 & 7.01 & 43.96 & 16.26 & 41.18 & 2753.3 & 404.0 \\
\hline $\mathrm{N}_{3} \mathrm{C}_{0}$ & 45.76 & 23.86 & 1.82 & 1.06 & 5.71 & 42.78 & 0.00 & 39.67 & 2893.2 & 395.0 \\
\hline $\mathrm{N}_{3} \mathrm{C}_{1}$ & 33.86 & 19.83 & 1.80 & 1.25 & 6.17 & 47.07 & 7.53 & 41.37 & 3072.9 & 441.0 \\
\hline $\mathrm{N}_{3} \mathrm{C}_{2}$ & 31.15 & 18.48 & 1.79 & 1.37 & 6.78 & 46.95 & 12.42 & 40.75 & 3045.8 & 433.0 \\
\hline $\mathrm{N}_{3} \mathrm{C}_{3}$ & 30.92 & 17.75 & 1.75 & 1.49 & 7.19 & 44.84 & 17.77 & 40.48 & 2999.5 & 428.0 \\
\hline $\mathrm{N}_{4} \mathrm{C}_{0}$ & 45.16 & 23.51 & 1.76 & .99 & 5.58 & 44.72 & 0.00 & 39.01 & 2854.8 & 388.0 \\
\hline $\mathrm{N}_{4} \mathrm{C}_{1}$ & 32.91 & 19.23 & 1.76 & 1.15 & 6.02 & 49.00 & 6.71 & 40.93 & 3033.7 & 432.0 \\
\hline $\mathrm{N}_{4} \mathrm{C}_{2}$ & 30.42 & 17.43 & 1.74 & 1.27 & 6.62 & 48.29 & 11.53 & 40.23 & 3011.0 & 422.0 \\
\hline $\mathrm{N}_{4} \mathrm{C}_{3}$ & 30.19 & 16.97 & 1.69 & 1.39 & 7.15 & 46.93 & 17.29 & 39.77 & 2952.5 & 413.0 \\
\hline S. Em \pm & 0.43 & 0.30 & 0.03 & 0.01 & 0.02 & 0.34 & 0.19 & 0.36 & 23.74 & 5.40 \\
\hline $\begin{array}{l}\text { C.D. at } \\
5 \%\end{array}$ & 1.21 & 0.83 & 0.07 & 0.04 & 0.06 & 0.95 & 0.53 & 1.01 & 66.65 & 15.10 \\
\hline
\end{tabular}

Treatments: Nitrogen levels- $\mathrm{N}_{0}, 0 \mathrm{~kg} \mathrm{~N} / \mathrm{ha} ; \mathrm{N}_{1}, 50 \mathrm{~kg} \mathrm{~N} / \mathrm{ha} ; \mathrm{N}_{2}, 100 \mathrm{~kg} \mathrm{~N} / \mathrm{ha} ; \mathrm{N}_{3}, 150 \mathrm{~kg} \mathrm{~N} / \mathrm{ha} ; \mathrm{N}_{4}, 200 \mathrm{~kg} \mathrm{~N} / \mathrm{ha}$ and cutting frequencies- $C_{0}$, no cutting; $C_{1}$, one cutting at 45 DAP; $C_{2}$, two cuttings at 45 and 65 DAP; $C_{3}$, three cuttings at 45,65 and 85 DAP. 
Table 2. Effect of cutting frequencies and nitrogen levels on seed yield and its quality parameters of water spinach (pooled).

\begin{tabular}{|c|c|c|c|c|c|c|c|c|}
\hline Treatment & $\begin{array}{c}\text { No. of } \\
\text { flowers / } \\
\text { hill } \\
\end{array}$ & $\begin{array}{c}\text { No. of } \\
\text { capsules / } \\
\text { hill }\end{array}$ & $\begin{array}{c}\text { Seed yield } \\
(\mathrm{t} / \mathrm{ha})\end{array}$ & $\begin{array}{c}\text { Shelling } \\
\text { percentage }\end{array}$ & $\begin{array}{l}1000 \text { seed } \\
\text { weight }(g)\end{array}$ & $\begin{array}{c}\text { Germination } \\
\text { percentage }\end{array}$ & $\begin{array}{c}\text { Seedling } \\
\text { vigour } \\
\text { index }\end{array}$ & $\begin{array}{c}\text { Seedling } \\
\text { growth } \\
\text { rate }\end{array}$ \\
\hline \multicolumn{9}{|c|}{ Cutting frequency } \\
\hline $\mathrm{C}_{0}$ & 274.3 & 210.6 & 1.17 & 61.14 & 39.84 & $82.7(9.09)$ & 5.24 & 0.106 \\
\hline $\mathrm{C}_{1}$ & 257.3 & 193.2 & 0.93 & 59.72 & 38.24 & $79.7(8.93)$ & 5.00 & 0.103 \\
\hline $\mathrm{C}_{2}$ & 239.3 & 179.3 & 0.81 & 58.97 & 37.25 & $77.3(8.79)$ & 4.86 & 0.100 \\
\hline $\mathrm{C}_{3}$ & 206.8 & 156.9 & 0.69 & 57.61 & 35.94 & $75.0(8.66)$ & 4.63 & 0.096 \\
\hline S. Em \pm & 0.73 & 0.77 & 0.01 & 0.34 & 0.25 & 0.30 & 0.04 & 0.001 \\
\hline C.D. at $5 \%$ & 2.06 & 2.16 & 0.01 & 0.95 & 0.70 & 0.85 & 0.14 & 0.003 \\
\hline \multicolumn{9}{|c|}{ Nitrogen level } \\
\hline $\mathrm{N}_{0}$ & 208.1 & 140.6 & 0.75 & 54.58 & 35.22 & $73.7(8.58)$ & 4.37 & 0.081 \\
\hline $\mathrm{N}_{1}$ & 231.6 & 169.2 & 0.83 & 57.31 & 36.37 & $76.2(8.73)$ & 4.65 & 0.088 \\
\hline $\mathrm{N}_{2}$ & 249.0 & 191.1 & 0.92 & 60.36 & 38.19 & $78.9(8.88)$ & 4.94 & 0.102 \\
\hline $\mathrm{N}_{3}$ & 272.2 & 218.4 & 1.02 & 62.99 & 40.13 & $83.5(9.14)$ & 5.44 & 0.121 \\
\hline $\mathrm{N}_{4}$ & 261.3 & 205.6 & 0.98 & 61.56 & 39.16 & $81.2(9.01)$ & 5.27 & 0.116 \\
\hline S. Em \pm & 0.82 & 0.86 & 0.01 & 0.38 & 0.28 & 0.34 & 0.05 & 0.001 \\
\hline C.D. at $5 \%$ & 2.30 & 2.42 & 0.02 & 1.06 & 0.78 & 0.95 & 0.15 & 0.004 \\
\hline \multicolumn{9}{|c|}{ Interaction (C X N) } \\
\hline $\mathrm{N}_{0} \mathrm{C}_{0}$ & 231.0 & 159.2 & 0.98 & 55.98 & 37.28 & 77.2 & 4.68 & 0.086 \\
\hline $\mathrm{N}_{0} \mathrm{C}_{1}$ & 213.0 & 144.4 & 0.82 & 54.57 & 35.26 & 74.7 & 4.41 & 0.083 \\
\hline $\mathrm{N}_{0} \mathrm{C}_{2}$ & 203.4 & 135.4 & 0.67 & 54.15 & 34.67 & 72.1 & 4.30 & 0.079 \\
\hline $\mathrm{N}_{0} \mathrm{C}_{3}$ & 185.0 & 123.4 & 0.56 & 53.64 & 33.68 & 70.7 & 4.09 & 0.075 \\
\hline $\mathrm{N}_{1} \mathrm{C}_{0}$ & 256.7 & 19.1 & 1.13 & 59.25 & 38.77 & 8.0 & 4.99 & 0.092 \\
\hline $\mathrm{N}_{1} \mathrm{C}_{1}$ & 245.5 & 177.8 & 0.85 & 57.92 & 36.65 & 77.3 & 4.77 & 0.090 \\
\hline $\mathrm{N}_{1} \mathrm{C}_{2}$ & 229.0 & 165.5 & 0.74 & 57.45 & 35.69 & 74.9 & 4.56 & 0.087 \\
\hline $\mathrm{N}_{1} \mathrm{C}_{3}$ & 195.1 & 143.7 & 0.62 & 54.62 & 34.40 & 72.5 & 4.27 & 0.083 \\
\hline $\mathrm{N}_{2} \mathrm{C}_{0}$ & 279.9 & 217.7 & 1.20 & 62.10 & 40.40 & 83.2 & 5.26 & 0.105 \\
\hline $\mathrm{N}_{2} \mathrm{C}_{1}$ & 263.7 & 200.4 & 0.94 & 60.84 & 38.70 & 79.7 & 4.97 & 0.103 \\
\hline $\mathrm{N}_{2} \mathrm{C}_{2}$ & 244.1 & 185.6 & 0.83 & 59.64 & 37.62 & 77.7 & 4.87 & 0.100 \\
\hline $\mathrm{N}_{2} \mathrm{C}_{3}$ & 208.3 & 160.8 & 0.70 & 58.86 & 36.06 & 75.2 & 4.69 & 0.099 \\
\hline $\mathrm{N}_{3} \mathrm{C}_{0}$ & 308.6 & 249.5 & 1.32 & 64.71 & 41.65 & 87.9 & 5.70 & 0.128 \\
\hline $\mathrm{N}_{3} \mathrm{C}_{1}$ & 287.7 & 229.6 & 1.09 & 63.62 & 40.69 & 84.5 & 5.52 & 0.124 \\
\hline $\mathrm{N}_{3} \mathrm{C}_{2}$ & 265.7 & 211.7 & 0.88 & 62.52 & 39.60 & 82.4 & 5.39 & 0.119 \\
\hline $\mathrm{N}_{3} \mathrm{C}_{3}$ & 226.9 & 183.0 & 0.82 & 61.13 & 38.59 & 79.3 & 5.16 & 0.112 \\
\hline $\mathrm{N}_{4} \mathrm{C}_{0}$ & 295.5 & 236.4 & 1.24 & 63.67 & 41.11 & 85.4 & 5.58 & 0.122 \\
\hline $\mathrm{N}_{4} \mathrm{C}_{1}$ & 276.4 & 214.1 & 098 & 61.65 & 39.88 & 82.5 & 5.36 & 0.119 \\
\hline $\mathrm{N}_{4} \mathrm{C}_{2}$ & 254.4 & 198.5 & 0.92 & 61.10 & 38.69 & 79.7 & 5.19 & 0.114 \\
\hline $\mathrm{N}_{4} \mathrm{C}_{3}$ & 218.8 & 173.5 & 0.77 & 59.82 & 36.97 & 77.2 & 4.94 & 0.110 \\
\hline S. Em \pm & 1.64 & 1.72 & 0.01 & 0.76 & 0.56 & 0.68 & 0.10 & 0.003 \\
\hline C.D. at $5 \%$ & 4.60 & 4.83 & 0.03 & 2.12 & 1.57 & 1.90 & 0.27 & 0.007 \\
\hline
\end{tabular}

Treatments: Nitrogen levels- $\mathrm{N}_{0}, 0 \mathrm{~kg} \mathrm{~N} / \mathrm{ha} ; \mathrm{N}_{1}, 50 \mathrm{~kg} \mathrm{~N} / \mathrm{ha} ; \mathrm{N}_{2}, 100 \mathrm{~kg} \mathrm{~N} / \mathrm{ha} ; \mathrm{N}_{3}, 150 \mathrm{~kg} \mathrm{~N} / \mathrm{ha} ; \mathrm{N}_{4}, 200 \mathrm{~kg} \mathrm{~N} / \mathrm{ha}$ and cutting frequencies- $C_{0}$, no cutting; $C_{1}$, one cutting at 45 DAP; $C_{2}$, two cuttings at 45 and 65 DAP; $C_{3}$, three cuttings at 45,65 and 85 DAP, *-Figures in the parenthesis indicate square root transformed values.

present investigation.

Significant variations on shelling percentage, 1000 seed weight, germination percentage, seedling vigour index (SVI) and seedling growth rate (Table 2) were noticed due to both the treatments and also their interaction. These quality parameters showed gradual 
Table 3. Economics of water spinach green and seed production for different cutting frequencies and nitrogen levels.

\begin{tabular}{ccccccc}
\hline $\begin{array}{c}\text { Treatment } \\
\text { combinations }\end{array}$ & $\begin{array}{c}\text { Total green } \\
\text { yield (t/ha) }\end{array}$ & $\begin{array}{c}\text { Total seed } \\
\text { yield (t/ha) }\end{array}$ & $\begin{array}{c}\text { Total input } \\
\text { (Rs.) }\end{array}$ & $\begin{array}{c}\text { Gross return } \\
\text { (Rs.) }\end{array}$ & $\begin{array}{c}\text { Net return } \\
\text { (Rs.) }\end{array}$ & BCR \\
\hline $\mathrm{C}_{0} \mathrm{~N}_{0}$ & - & 0.98 & 46296 & 107800 & 61504 & 2.33 \\
$\mathrm{C}_{0} \mathrm{~N}_{1}$ & - & 1.13 & 46841 & 124300 & 77459 & 2.65 \\
$\mathrm{C}_{0} \mathrm{~N}_{2}$ & - & 1.20 & 47381 & 132000 & 84619 & 2.79 \\
$\mathrm{C}_{0} \mathrm{~N}_{3}$ & - & 1.32 & 47926 & 145200 & 97274 & 3.03 \\
$\mathrm{C}_{0} \mathrm{~N}_{4}$ & - & 1.24 & 48466 & 136400 & 87934 & 2.81 \\
$\mathrm{C}_{1} \mathrm{~N}_{0}$ & 4.29 & 0.82 & 47454 & 108433 & 60979 & 2.29 \\
$\mathrm{C}_{1} \mathrm{~N}_{1}$ & 4.92 & 0.85 & 47999 & 114410 & 66411 & 2.38 \\
$\mathrm{C}_{1} \mathrm{~N}_{2}$ & 5.79 & 0.94 & 48539 & 128008 & 79469 & 2.64 \\
$\mathrm{C}_{1} \mathrm{~N}_{3}$ & 7.53 & 1.09 & 49084 & 151903 & 102819 & 3.09 \\
$\mathrm{C}_{1} \mathrm{~N}_{4}$ & 6.71 & 0.98 & 49624 & 136318 & 86694 & 2.75 \\
$\mathrm{C}_{2} \mathrm{~N}_{0}$ & 8.69 & 0.67 & 48612 & 110633 & 62021 & 2.28 \\
$\mathrm{C}_{2} \mathrm{~N}_{1}$ & 9.87 & 0.74 & 49157 & 123348 & 74191 & 2.51 \\
$\mathrm{C}_{2} \mathrm{~N}_{2}$ & 10.82 & 0.83 & 49697 & 137285 & 87588 & 2.76 \\
$\mathrm{C}_{2} \mathrm{~N}_{3}$ & 12.42 & 0.88 & 50242 & 149585 & 99343 & 2.98 \\
$\mathrm{C}_{2} \mathrm{~N}_{4}$ & 11.53 & 0.92 & 50782 & 150203 & 99421 & 2.96 \\
$\mathrm{C}_{3} \mathrm{~N}_{0}$ & 14.83 & 0.56 & 49769 & 124628 & 74859 & 2.50 \\
$\mathrm{C}_{3} \mathrm{~N}_{1}$ & 15.57 & 0.62 & 50314 & 134373 & 84059 & 2.67 \\
$\mathrm{C}_{3} \mathrm{~N}_{2}$ & 16.26 & 0.70 & 50854 & 146105 & 95251 & 2.87 \\
$\mathrm{C}_{3} \mathrm{~N}_{3}$ & 17.77 & 0.82 & 51399 & 165723 & 114324 & 3.22 \\
$\mathrm{C}_{3} \mathrm{~N}_{4}$ & 17.29 & 0.77 & 51939 & 158183 & 106244 & 3.05 \\
\hline
\end{tabular}

Sale price of green produce Rs. 4.00 per kg; seed, Rs. 110.00 per kg.

decrease with higher cutting frequency but reverse results were seen due to $\mathrm{N}$ fertilization upto $150 \mathrm{~kg} \mathrm{~N} /$ ha. Significant variations on shelling percentage, 1000 seed weight, germination percentage, seedling vigour index (SVI) and seedling growth rate (Table 2) were noticed due to both the treatments and also their interaction. These quality parameters showed gradual decrease with higher cutting frequency but reverse results were seen due to $\mathrm{N}$ fertilization upto $150 \mathrm{~kg} \mathrm{~N} /$ ha. Significantly maximum shelling percentage (61.14), 1000 seed weight $(39.84 \mathrm{~g})$, germination percentage (82.7), SVI (5.24) and seedling growth rate $(0.106 \mathrm{~g} / \mathrm{plant} /$ day $)$ were recorded in control treatment $\left(\mathrm{C}_{0}\right)$ where plants were kept untouched. On the contrary the highest cutting treatment of three cuttings $\left(\mathrm{C}_{3}\right)$ recorded minimum of all these parameters. Pandita and Randhawa (1996) reported that cutting of leaves significantly reduced 1000 seed weight and germination percentage in spinach beet. The application of $150 \mathrm{~kg} \mathrm{~N} / \mathrm{ha}\left(\mathrm{N}_{3}\right)$ gave significantly superior quality seed of water spinach (shelling percentage, 62.99; 1000 seed weight, $40.13 \mathrm{~g}$; germination percentage, 83.5; SVI, 5.44 and seedling growth rate, $0.121 \mathrm{~g} / \mathrm{plant} /$ day) closely followed by $\mathrm{N}_{4}$ (200 kg N/ha) and $\mathrm{N}_{2}$ treatment (100 kg N/ha) and comparatively poor quality seed was recorded in control $\left(\mathrm{N}_{0}\right)$. The combination of no cutting with 150 $\mathrm{kg} \mathrm{N} / \mathrm{ha}$ application $\left(\mathrm{C}_{0} \mathrm{~N}_{3}\right)$ showed best quality water spinach seed with respect to all parameters such as shelling percentage (64.71), 1000 seed weight (41.65 g), germination percentage (87.9), SVI (5.70) and seedling growth rate $(0.128 \mathrm{~g} / \mathrm{plant} /$ day $)$. This was closely followed by $\mathrm{C}_{0} \mathrm{~N}_{4}, \quad \mathrm{C}_{1} \mathrm{~N}_{3}$ and $\mathrm{C}_{2} \mathrm{~N}_{3}$ combinations. Improvement in all these seed quality attributes because of the greater supply of nitrogen might be due to the fact that nitrogen helps in protein synthesis and ultimately increase the plumpness of seed. Effect of nitrogen on yield and yield attributes of fenugreek was reported by Sharma (2000). Present findings in this study were in conformity with the results by Sharma (2000). The economics of production for water spinach cultivation under cutting frequencies and nitrogen levels (Table 3 ) revealed that the gross return and net return of water spinach was influenced to a great extend by combination of cutting frequencies and nitrogen levels. The combination of three cutting with $150 \mathrm{~kg} \mathrm{~N} / \mathrm{ha}\left(\mathrm{C}_{3} \mathrm{~N}_{3}\right)$ recorded maximum gross return (Rs. 1,65,723.00), highest net return (Rs. 1,14,324.00) as well as highest B:C ratio (2.22). Comparatively, higher $\mathrm{B}: \mathrm{C}$ ratio was also recorded in $\mathrm{C}_{1} \mathrm{~N}_{3}$ (2.09), $\mathrm{C}_{3} \mathrm{~N}_{4}$ (2.05) and $\mathrm{C}_{0} \mathrm{~N}_{3}$ (2.03). The treatment combination $\mathrm{C}_{2} \mathrm{~N}_{0}$ recorded lowest $\mathrm{B}$ :C ratio (1.28) amongst all treatments combinations.

Maximum green yield and seed yield of the upland water spinach crop was recorded with the treatment combination of three cuttings with $150 \mathrm{~kg} \mathrm{~N} / \mathrm{ha}$ and no cutting with $150 \mathrm{~kg} \mathrm{~N} / \mathrm{ha}$. Considering the economics of the crop cultivation, the treatment combination of 
three cuttings with $150 \mathrm{~kg} \mathrm{~N} / \mathrm{ha}$ that gave $17.77 \mathrm{t} / \mathrm{ha}$ green and 0.82 t/ha seed yield was the higher remunerative and may be recommended under terai zone of West Bengal.

\section{Conclusion}

It was concluded that on economic point of view, the combination of three cuttings and $150 \mathrm{~kg}$ N/ha was noticed to be superior amongst all other remaining treatment combinations with respect to growth, yield, and economic point of view and that may be adopted for its commercial cultivation in medium to upland situation under terai region of West Bengal.

\section{REFERENCES}

Ahmed, M. and Oladiaran, J.A. (2012). Effect of stem cutting and variety on shoot development and seed yield of jute mallow (Corchorus olitorious L.). Experimental Agriculture and Horticulture, 12(3): 21-29.

Datta, S., Alam, K. and Chatterjee, R. (2005). Effect of different levels of nitrogen and leaf cutting on leaf and seed yield of fenugreek (Trigonella foenum graecum ). Indian Journal of Agricultural. Sciences,75(9): 580581.

Datta, S., Alam, K. and Chatterjee, R. (2008). Effect of different levels of nitrogen and leaf cutting on growth, leaf and seed yield of coriander. Indian Journal of Horticulture, 65 (2): 201-203.

Gomez, K.A. and Gomez, A.A. (1984). Statistical Procedures in Agricultural Research, Chichester 2nd edition, New York. pp. 680.

Jana, J.C., Thapa, U. and Maity, T.K. (1999). Green and seed yield of palak (Beta vulgaris L. var. bengalensis hort.) as affected by nitrogen fertilization and cutting management. Vegetable Science, 26 (1): 61-63.

Korus, A.A. and Lisiewska, Z. (2009). Effect of cultivar and harvest date of kale (Brassica oleracea L var. Acephala). Polish Journal of Environment Study, 18(2): 235-241.

Li, L. and Harahap A.D. (1994). Nitrogen effect on water convolvulus. ARC Training pp. 24-67.
Masinde, P.W. and Agong, S.G. (2012). The influence of nitrogen application on growth and mineral content of two African night shade species (Solanum spp.) cultivated in Kenya. JAGST, 14(2): 44.

Palada, M.C. and Crossman, S.M. A. (1999). Planting density affects growth and yield of bush okra (Corchorus olitorius). Proceedings of 34th Annual Meeting 1998 - Caribbean Food Crop Society, pp. 52-57.

Pandita, V.K. and Randhawa, K.S. (1996). Effect of seed size and leaf cutting on seed productivity and quality in beet leaf. Seed Research, 24(2): 156-157.

Ranganna, S. (2001). Analysis and quality control for fruits and vegetable products. Tata Mc Grawhill Publication, New Delhi.

Schmidt, D.R., MacDonald, H.A. and Brockman, F.E. (2007). Oxalate and nitrate contents of four tropical leafy vegetables grown at two soil fertility levels. Journal of the Science of Food and Agriculture, 88(1): 723.

Sharma, S.K. (2000). Response of nitrogen and spacing on fenugreek seed production. Horticultural Journal, 13 (2): 39-42.

Singh, U.C., Sundararajan, S. and Veeraragavathatham, D. (1986). Effect of split application of nitrogen on crude protein, carotene and ascorbic acid contents of clipping amaranthus (Amaranthus tristis L.) cv. CO3. South Indian Horticulture, 34(3): 150-153.

Tehelan, S.K. and Thakral, K.K. (2008). Effect of different levels of nitrogen and leaf cutting on leaf and seed yield of coriander (Coriandrum sativum L.). Journal of Spices and Aromatic Crops, 17(2): 180-182.

Thapa, U. and Maity, T.K. (2004). Growth and yield of Basella spp. as influenced by nitrogen and phosphorus fertilization with or without cutting treatments. Crop Research, 28 (1, 2 and 3): 123-126.

Tomar, K.S. (2001). Effect of phosphorus, magnesium levels and cutting on the yield and chemical composition of spinach. Advances in Plant Sciences, 14 (2): 471-474.

Tunctruk, P., Colen, A.E., Tunctrunk, M. (2011). The effect of nitrogen and sulphur fertilizers on the yield and quality of fenugreek (Trigonella foenum graecum L.). Turkish Journal of Field Crops, 16(10): 69-75. 